\title{
The limit states of polymetallic plates with piecewise elliptic contours
}

\author{
Yuri Nemirovskii ${ }^{1, *}$ \\ ${ }^{1}$ Khristianovich Institute of Theoretical and Applied Mechanics Siberian Branch of the Russian \\ Academy of Sciences, Physics of Fast Processes Laboratory, 630090 Institutskaya str., 4/1, \\ Novosibirsk, Russia
}

\begin{abstract}
A unified approach to the study of plastic deformation of polymetallic isotropic plates with smooth and piecewise smooth support contours under the influence of static loads is developed. Plates of various types are considered: elliptical; semi-and quarter-elliptical; elliptical belt, «elliptical» sector. Materials of the layers can be ideally plastic or linear hardening. The first limit loads for ideally plastic materials and the second limit loads (initial destruction loads) for hardening materials are determined.
\end{abstract}

\section{Introduction}

The work is devoted to the study of limit states and optimal designing of round and ring plates made of ideal rigid plastic material. Exact analytical solutions under axisymmetric loading and fastening conditions are obtained from the Tresca plasticity condition $[1,2]$. The solution of similar problems under the Mises plasticity condition leads to more complex differential equations with features and uncertainties that must be disclosed in solution process and therefore can be completed in rare cases [3,4]. The ap-proximate solutions for plates of other geometric forms are usually obtained using the kinematic Gvozdev-Yogansen method [5]. In recent decades, the intensive researches have been carried out to create the effective polymetallic structures for various industries and the construction industry. From the technological point of view, the easiest methods of manufacturing are gluing, explosion welding, diffusion welding, cold gas dynamic and plasma spraying, which allow to create the layered packages from almost any set of materials on large areas, and then to obtain from them the laminated plates of required forms by laser cutting. The problem of determining the first limit state of laminated plates and the rational selection of materials for flat barriers was considered in [6] within a model of ideal rig-id plastic materials. However, many metal materials and alloys have the property of significant hardening, and the load limit of a non-metallic structure may be too underestimated in fact. In this regard, for such structures it was proposed to introduce a second limit state [7] in which, according to the concept of the weakest link any of the materials of layered package reached the limit deformation of prefracture. The

\footnotetext{
* Corresponding author: strangcheb@mail.ru
} 
corresponding solutions for round and ring plates were considered in [8]. In this paper, we produced a uniform approach for determining the first and second limit loads for various shapes of plates, formed from an elliptical plate by cutting its various parts off (semiellipses, quarter-ellipses, elliptical sectors and belts).

\section{Problem statement}

We consider a polymetallic plate from a set of materials arranged in pairs symmetrically with respect to the reference (middle) plane $z=0$. Let $h_{j}$ denote the coordinates of the section of layers in direction of the normal to the reference surface:

$$
0 \leq z \leq h_{1} \leq h_{2} \leq h_{j} \leq h_{n}, h_{i}-h_{i-1}=\Delta_{i} .
$$

In the case of transverse bending of the plate at rigid joining of layers in accordance with the Kirchhoff-Lyav hypotheses for the deformation components, we have expressions:

$$
\begin{aligned}
& \varepsilon_{x}=-z \chi_{x}, \varepsilon_{y}=-z \chi_{y}, \varepsilon_{x y}=-z \chi_{x y}, \\
& \chi_{x}=\frac{\partial^{2} \omega}{\partial x^{2}}, \chi_{y}=\frac{\partial^{2} \omega}{\partial y^{2}}, \chi_{x y}=\frac{\partial^{2} \omega}{\partial x \partial y},
\end{aligned}
$$

where $\omega$ is deflection of the reference plane.

We will consider the quasistatic loads of high intensity in which the elastic deformations will be significantly lower than plastic. Then the connection between stresses and deformations for the $i$-material can be used in the form of relations of the theory of Genka Ilyushin [8]:

$$
\begin{gathered}
\sigma_{x i}=\frac{\Phi_{i}\left(e_{u}\right)}{e_{u}}\left(\varepsilon_{x}+\frac{1}{2} \varepsilon_{y}\right), \Phi_{i}\left(e_{u}\right)=\sigma_{0 i}+B_{i} e_{u}, \\
\sigma_{y i}=\frac{\Phi_{i}\left(e_{u}\right)}{e_{i}}\left(\frac{1}{2} \varepsilon_{x}+\varepsilon_{y}\right), \tau_{i}=\frac{\Phi_{i}\left(e_{u}\right)}{e_{u}} \varepsilon_{x y}, \\
M_{x y}=D_{1} J_{5}(\omega)+D_{2} J_{6}(\omega),
\end{gathered}
$$

where

$$
\begin{gathered}
D_{1}=\sum_{i=1}^{n} \sigma_{0 i}\left(h_{i}^{2}-h_{i-1}^{2}\right), D_{2}=\frac{2}{3} \sum_{i=1}^{n} B_{i}\left(h_{i}^{3}-h_{i-1}^{3}\right) \\
J_{1}(\omega)=\frac{\chi_{x}+1 / 2 \chi_{y}}{\chi_{u}}, J_{2}(\omega)=\chi_{x}+1 / 2 \chi_{y}, \\
J_{3}(\omega)=\frac{\chi_{y}+1 / 2 \chi_{x}}{\chi_{u}}, J_{4}(\omega)=\chi_{y}+1 / 2 \chi_{x}, \\
J_{5}(\omega)=\frac{\chi_{x y}}{\chi_{u}}, J_{6}(\omega)=\chi_{x y} .
\end{gathered}
$$

For plates lying on elastic base with a resistance coefficient $k_{1}$, the equilibrium equations at bending have the form:

$$
\frac{\partial^{2} M_{x}}{\partial x^{2}}+2 \frac{\partial^{2} M_{x y}}{\partial x \partial y}+\frac{\partial^{2} M_{y}}{\partial y^{2}}-k_{1} \omega+q_{0} \varphi(x, y) \cdot=0
$$

where $q_{0}$ is amplitude of the external load, $\varphi(x, y)$ is the law of its distribution on the plate surface. form:

Substituting the expression (3) into equations (6), we can represent it in the operator 


$$
R(\omega)-k_{1} \omega-q_{0} \varphi(x, y)=0 .
$$

Consider a class of plates of different shapes, which contours are formed from an elliptical plate with a contour, described by the equation (Figure 1):

$$
\frac{x^{2}}{a^{2}}+\frac{y^{2}}{b^{2}}=1
$$

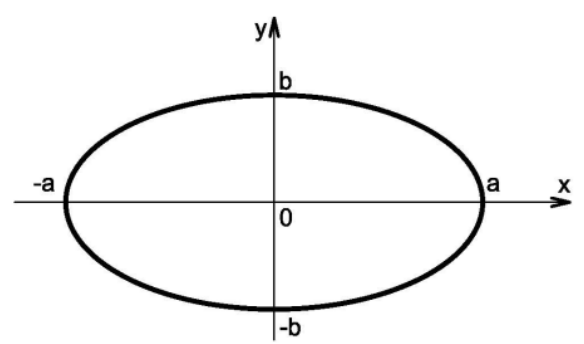

Fig. 1. Elliptical plate with a contour, described by the equation (8).

Plates of other shapes are formed by cutting off some parts from the elliptical plate. In particular, we consider semi-elliptic plates (Figure 2a, 2b):

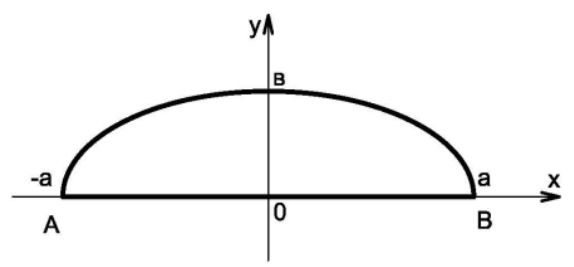

Fig. 2a. Semi-elliptic plate.

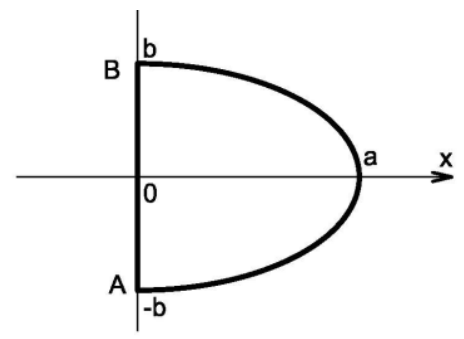

Fig. 2b. Semi-elliptic plate.

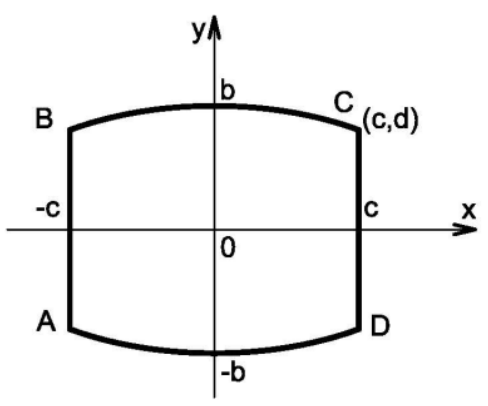

Fig. 3a. Elliptical belt. 


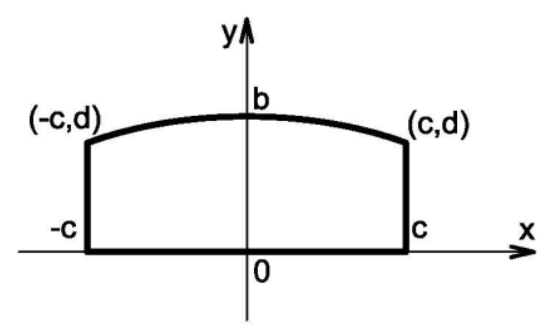

Fig. 3b. Quarter-belt.

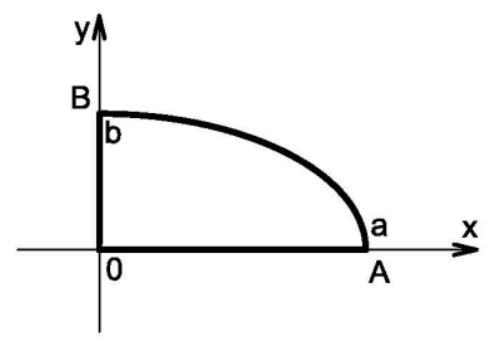

Fig. 4a. Quarter-belt.

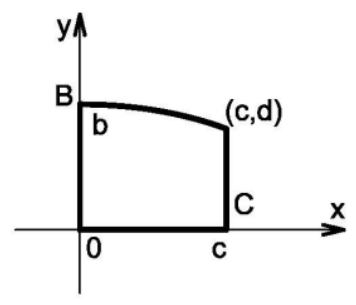

Fig. 4b. Quarter-belt.

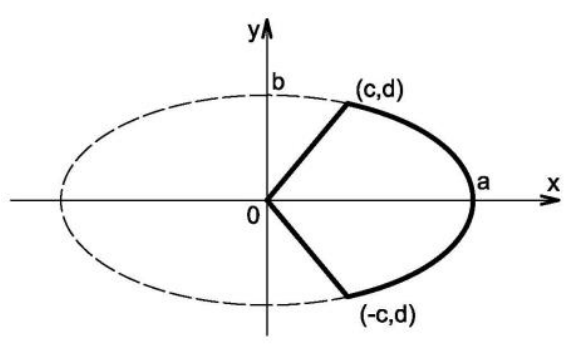

Fig. 5. Elliptical sector.

We assume that all new formed plates will be pinched along the contour or leant by hinges in some parts.

For all considered plates, we seek the approximate solution in the form of:

$$
\omega(x, y, t)=C f(x, y),
$$

where the function $f(x, y)$ is selected under the requirement of the boundary conditions of fastening on the contour of corresponding plate.

First, we consider the plates clamped along the entire contour. 
Then, under the condition of pinching the elliptical contour in Figure 1, we obviously have:

$$
\begin{gathered}
f(x, y)=\left(\frac{x^{2}}{a^{2}}+\frac{y^{2}}{b^{2}}-1\right)^{2}, \\
-a \leq x \leq a,-b \leq y \leq b .
\end{gathered}
$$

For the plate in Figure 2a we have:

$$
\begin{aligned}
& f(x, y)=\left(\frac{x^{2}}{a^{2}}+\frac{y^{2}}{b^{2}}-1\right)^{2} y^{2}, \\
& -a \leq x \leq a, 0 \leq y \leq b .
\end{aligned}
$$

For the plate in Figure $2 b$ we have:

$$
\begin{gathered}
f(x, y)=\left(\frac{x^{2}}{a^{2}}+\frac{y^{2}}{b^{2}}-1\right)^{2} x^{2}, \\
0 \leq x \leq a,-b \leq y \leq b .
\end{gathered}
$$

For the plate in Figure 3a:

$$
\begin{gathered}
f(x, y)=\left(\frac{x^{2}}{a^{2}}+\frac{y^{2}}{b^{2}}-1\right)^{2}\left(x^{2}-c^{2}\right)^{2}, \\
-c \leq x \leq c,-b \leq y \leq b .
\end{gathered}
$$

For the plate in Figure 3b:

$$
\begin{aligned}
f(x, y) & =\left(\frac{x^{2}}{a^{2}}+\frac{y^{2}}{b^{2}}-1\right)^{2}\left(x^{2}-c^{2}\right)^{2} y^{2}, \\
& -c \leq x \leq c, 0 \leq y \leq b .
\end{aligned}
$$

For the plate in Figure 4a:

$$
\begin{gathered}
f(x, y)=\left(\frac{x^{2}}{a^{2}}+\frac{y^{2}}{b^{2}}-1\right)^{2} x^{2} y^{2}, \\
0 \leq x \leq a, 0 \leq y \leq b
\end{gathered}
$$

For the plate in Figure 4b:

$$
\begin{gathered}
f(x, y)=\left(\frac{x^{2}}{a^{2}}+\frac{y^{2}}{b^{2}}-1\right)^{2}(x-c)^{2} x^{2} y^{2}, \\
0 \leq x \leq c, 0 \leq y \leq b .
\end{gathered}
$$

For the plate in Figure 5 we have:

$$
\begin{aligned}
f(x, y) & =\left(y^{2}-k_{1}^{2} x^{2}\right)^{2}\left(\frac{x^{2}}{a^{2}}+\frac{y^{2}}{b^{2}}-1\right)^{2}, \\
0 & \leq x \leq a,-d \leq y \leq d
\end{aligned}
$$

If the curvilinear parts of the contour are clamped, and the rectilinear ones are hinged, it is easy to select the functions $f(x, y)$ that satisfy these fastening conditions. For example, instead of functions (11) and (12), for the plates shown in Figure 2a, 2b we should use the functions: 


$$
\begin{aligned}
& f(x, y)=\left(\frac{x^{2}}{a^{2}}+\frac{y^{2}}{b^{2}}-1\right)^{2} y^{3}, \\
& -a \leq x \leq a, 0 \leq y \leq b, \\
& f(x, y)=\left(\frac{x^{2}}{a^{2}}+\frac{y^{2}}{b^{2}}-1\right)^{2} x^{3}, \\
& 0 \leq x \leq a,-b \leq y \leq b,
\end{aligned}
$$

And for the plates in Figure $3 \mathrm{a}$ and Figure $3 \mathrm{~b}$ we use functions:

$$
\begin{aligned}
& f(x, y)=\left(\frac{x^{2}}{a^{2}}+\frac{y^{2}}{b^{2}}-1\right)^{2}\left(x^{2}-c^{2}\right)^{3}, \\
& f(x, y)=\left(\frac{x^{2}}{a^{2}}+\frac{y^{2}}{b^{2}}-1\right)^{2}\left(x^{2}-c^{2}\right)^{3} y^{3} .
\end{aligned}
$$

The relation between the deflection $C$ and load $q_{0}$ amplitudes for the corresponding plate shape will be determined from the equation:

$$
\iint_{S}\left[R(\omega)-k_{1} \omega-q_{0} \varphi(x, y)\right] f(x, y) d x d y=0,
$$

where $S$ is the plate area.

If all materials of the layers are perfectly plastic $\left(B_{1}=B_{2}=\ldots=B_{n}=0\right)$, then if $C \rightarrow 0$, from the equation (22) we obtain the amplitude of the first limit load $q_{0}^{0}$. We need to add the equation to obtain the second limit load of pre-destruction $q_{0}^{*}$ :

$$
h_{k} \chi_{u}^{*}=\varepsilon_{k}^{*},
$$

where $\varepsilon_{k}^{*}$ is maximum permissible deformation of predestruction of material of $k$-layer («the weakest link ») and

$$
\chi_{u}^{*}=\max _{x, y \in S} \chi_{u}(x, y) .
$$

This work is carried out with the partial financial support of RFBR grant (project №17-41-210272).

\section{References}

1. J. N. Goodier, P. G. Hodge, Elasticity and plasticity (Moscow, 1960)

2. W. Freiberger, B. Tekinalp, Journal of Mechanics and Physics Solids, 4, 294-299 (1956)

3. A. A. Ilyushin, Plasticity (1946)

4. I. T. Vohnyanin, Y. V. Nemirovskii, Bulletin of the Yakovlev Chuvash state pedagogical university. Series: mechanics of limit state, 2, 85-96 (2014)

5. Y. V. Nemirovskii, T. P. Romanova, Dynamics of resistance of flat plastic barriers (Novosibirsk, 2009)

6. Y. V. Nemirovskii Conf. Proc. Modern methods of mathematical modelling of natural and anthropogenic disasters, 1, 191-194 (2003)

7. Y. V. Nemirovskii, Bulletin of the Yakovlev Chuvash state pedagogical university. Series: mechanics of limit state, 3, 150-159 (2009)

8. Y. V. Nemirovskii, Bulletin of the Yakovlev Chuvash state pedagogical university. Series: mechanics of limit state, 1, 189-195 (2015) 